NASA Technical Memorandum 86350

NASA-TM-86350 19850008553

\title{
STUDY OF WINGLETS APPLIED TO BIPLANES
}

Peter D. Gall and Hubert C. Smith

January 1985

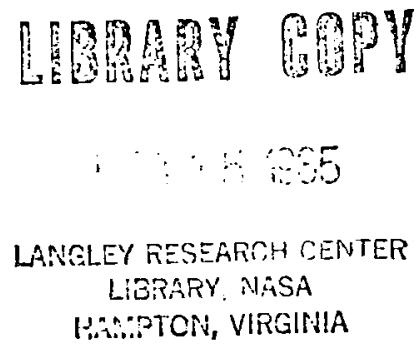

National Aeronautics and

Space Administration 
31176013087532 


\section{SUMMARY}

This paper examines, both theoretically and experimentally, the possibility of improving the aerodynamic characteristics of a biplane configuration by adding winglets. Theoretical calculations show good agreement with experiment in predicting inviscid drag due to lift. Theoretical and experimental results indicate that the addition of winglets to an optimized biplane configuration can increase the ideal efficiency factor by up to 13 percent, as well as increasing the lift-curve slope and maximum lift coefficient. A theoretical analysis comparing the biplane with an optimized winglet to an equivalent monoplane indicates that the biplane has the potential for a 6.4-percent increase in $L / D_{\max }$ and 13-percent increase in $C_{L} 3 / 2 / C_{D}$, the classical endurance parameter.

\section{INTRODUCTION}

The high cost of aviation fuel, coupled with increasing manufacturing and operating costs, is forcing aircraft manufacturers to build faster, more efficient aircraft. Considerable research has been conducted in government, industry, and universities in the areas of advanced aerodynamics. One such area is the study of winglets and wing-tip extensions. Research by NASA has determined that properly designed winglets can significantly reduce induced drag at cruise-lift coefficients without imposing severe additional structural loads (refs. 1 and 2). The presence of the winglet causes a physical constraint to the flow field at the location of the winglet, which is usually at or near the wing tip. This constraint weakens the strength of the trailing vorticity shed near the outboard section of the wing, resulting in a corresponding reduction in the induced downwash. By reducing the induced downwash, the effective angle of attack of the outboard section of the wing is increased. This results in a more even spanwise load distribution across the entire wing, resulting in greater induced efficiency.

Another area of recent research is the biplane wing or dual-wing concept. Biplanes have certain advantages, such as allowing a low wing loading while retaining light structural weight, resulting in a high lift at low speeds with rudimentary or no high-lift devices. Biplanes also offer good roll characteristics due to the smaller span and the lower inertia about the longitudinal axis. Because of the good roll characteristics, less aileron area is required, leaving more trailing edge space available for high-lift devices.

Since the early days of biplane theory, it has been shown by Prandt 1 (ref . 3), Munk (ref. 4), and others that, for a given span, the biplane produces less induced drag. Many studies, both theoretical and experimental, have been made of the efficiency of biplanes having various combinations of gap, stagger, and decalage (ref. 5-8). Nenadovitch (ref. 9) and 01 son (ref. 10) discovered certain combinat ions that appeared to be nearly optimum for rectangular untwisted biplane wings. Numerical two-dimensional analysis by Rokhsaz (ref. 11) confirmed that the combinations of gap, stagger, and decalage determined by Nenadovitch do approach optimum arrangements. 
The purpose of the present study is to examine, both theoretically and experimentally, the possibility of further increasing the aerodynamic efficiency of a biplane configuration by adding winglets. The first step in the study was to produce experimental and theoretical data for a biplane configuration. The experimental data was obtained from wind-tunnel tests performed in the Pennsylvania State University subsonic wind tunnel at a Reynolds number of 510,000. The theoretical calculations were carried out using two vortex-lattice type potential flow codes. Then, the computed results were verified by comparison with the experimental data. Finally, the computer codes were used to develop an improved winglet for the biplane.

\section{SYMBOLS}

$\begin{array}{ll}\text { b } & \text { wing span } \\ c & \text { wing chord } \\ C_{D} & \text { total drag coefficient } \\ C_{D_{i}} & \text { induced drag coefficient } \\ \Delta C_{D} & \text { incremental profile drag coefficient } \\ C_{D_{\text {min }}} & \text { minimum drag coefficient } \\ C_{L} & \text { lift coefficient } \\ C_{L} 3 / 2 / C_{D} & \text { endurance parameter }\end{array}$

Dec incidence angle between upper and lower wings, positive when upper wing is at higher angle of attack than the lower wing

EMAR equivalent monoplane aspect ratio, $\frac{S}{2 c^{2}(1+\sigma)}$

e efficiency factor

Ga vertical distance between the two wings, nondimensionalized by chord

L/D lift-drag ratio

St longitudinal distance between the 50-percent mean chord points of the two wings, nondimensionalized by chord

$\alpha \quad$ angle of attack

o Prandt l's biplane interference factor 


\section{THEORETICAL ANALYSIS}

Theoretical predictions for the biplane configurations, both with and without winglets, were primarily carried out using a vortex-lattice type potential flow code developed by the author specifically for multi-wing winglet configurations (ref. 12). The code utilizes a vortex-lattice representation of the aircraft lifting surfaces, coupled with classic equations and theorems for computing such aerodynamic characteristics as lift, induced drag, spanwise and chordwise load distributions, and wing efficiency factors. The code assumes steady, irrotational, inviscid, incompressible attached flow. Therefore, numerical results can only be assumed valid at subsonic speed when the wing is operating at a less than critical angle of attack. Induced drag calculations are performed by applying Munk's theorems I and II to the wing induced flow field located an infinite distance downstream from the lifting surfaces in the Trefftz plane. Munk's first theorem states that the induced drag of a multiplane lifting system is unaltered if any of the lifting elements are moved in the direction of the motion provided that the attitude of the elements is adjusted to maintain the same distribution of $1 \mathrm{ift}$ among them. Munk's second theorem states that when calculating the induced drag of a lifting system, one may, instead of using the actual values of the velocity normal to the lifting elements at the original point of application of the forces, use one-half of the limiting value of the normal velocity for the corresponding values at the original points. This theorem allows the drag computations to be done in the Trefftz plane.

The second vortex-lattice code, which was used to verify the results of the first code, was a unified vortex-lattice code (NARUVL) developed by North American Rockwell (ref. 13). In both codes, the lifting surfaces were modeled with zero thickness airfoils.

\section{EXPERIMENTAL PROCEDURE}

Experimental results were obtained by testing a half-span wind-tunnel model of the biplane-winglet configuration in the Pennsylvania State University subsonic wind tunnel at a Reynolds number of 510,000 .

The model consisted of two identical wings, each having a chord of 8 inches and a semispan of 20 inches, yielding a full-span aspect ratio per wing of five. Figure 1 is an isometric view of the model. The airfoil used was a symmetrical NACA 0012 section. Spanning the tips of the two wings was a constant chord, 3-percent thick symmetrical airfoil winglet. The configuration with winglets may be thought of as a "box" wing. The model was constructed to have a fixed gap and stagger, both equal to one chord length, and a decalage angle that could be varied between 0 and -6 degrees.

The model was tested throughout the operating angle-of-attack range at decalage angles of 0 and -5 degrees. All configurations were tested with and without winglets.

Through calibration tests conduced on the balance, the following experimental accuracies were determined: $C_{D} \pm .0005, C_{L} \pm 0.02$, and $\alpha \pm 1^{\circ}$. 


\section{RESULTS AND DISCUSSION}

Theoretical and Experimental Results.- The purpose of obtaining the experimental data was to study the relative differences in induced drag for the various configurations, and then to compare these results to theory.

The results are presented for both $0^{\circ}$ and $-5^{\circ}$ decalage configurations. Figures 2 and 3 present $C_{L}$ as a function of $\alpha$ for the $0^{\circ}$ and $-5^{\circ}$ decalage configurations. For the $0^{\circ}$ decalage configuration, loss of lift begins to occur at an angle of attack of approximately $13^{\circ}$. This is probably due to stall onset of the upper wing. The vortex-lattice code predicts that at $\alpha=12^{0}$ the upper wing is at a $C_{L}$ of .870 , while the lower wing is at $a, C_{L}$ of only .614. This occurs since the lower wing is immersed in the induced downwash of the upper wing; hence, the lower wing operates at a less effective angle of attack. For the $-5^{\circ}$ decalage configuration, the lower wing is only slightly more highly loaded while approaching the stall, resulting in only a slight increase in $C_{L_{\max }}$ over the $0^{\circ}$ decalage configuration.

The addition of winglets to both configurations resulted in approximately a 5-percent increase in lift curve slope and a 4-percent increase in maximum lift coefficient. The maximum lift coefficient for the $0^{\circ}$ decalage configuration was .850 without winglets and .881 with winglets.

The magnitudes of the experimental data cannot be taken to be characteristic of full-scale designs, or of designs using new high-lift, low-drag airfoils. A much higher maximum lift coefficient could be expected at full-scale Reynolds numbers. For example, a NACA 0012 two-dimensional airfoil tested at a Reynolds number of 500,000 has $C_{L_{\max }}=0.900^{14}$; whereas the same airfoil tested at a typical full-scale Reynolds number of $6,000,000$ has $C_{L_{\max }}=1.600$.

At low Reynolds numbers, profile-drag coefficients can vary quite considerably with Reynolds number (ref. 14); therefore, it is important to know the effect of Reynolds numbers on profile drag when analyzing the drag data. The profile drag coefficient, which consists of skin-friction drag, pressure (form) drag, and interference drag, can be expressed as: $C_{D_{p}}=C_{D_{\text {min }}}+\Delta C_{D}$ where $\Delta C_{D}$ is the incremental change in profile drag due to lift. In a two-dimensional airfoil test, where there can be no induced drag, $\Delta C_{D_{p}}$ represents all the drag due to lift.

For the biplane-winglet configuration tested, it was necessary to determine the relationship between the incremental profile drag and the lift coefficient at the test Reynolds number of 510,000. This was done by studying the experimental results of reference 9, which were obtained from tests on a two-dimensional biplane configuration utilizing symmetrical airfoils.

Figures 4 and 5 show experimental and theoretical drag polars for both configurations. The symbols represent experimental data. The theoretical curves, which include viscous and inviscid drag, were computed from

$$
C_{D}=C_{D_{\text {min }}}+\Delta C_{D_{p}}+C_{D_{i}}
$$


Values of $C_{D_{\text {min }}}$ were obtained from the experimental data. $C_{D_{\min }}$ for the $0^{\circ}$ decalage no-winglet configuration was experimentally found to be .0150. At a fullscale Reynolds number of $6,000,000, C_{D_{\text {min }}}$ would be only 0.007 .15 The experimental data also showed that the presence of the winglets increased $C_{D_{\text {min }}}$ by 15-20 counts. Values for $\Delta C_{D_{p}}$ were obtained from reference 9. The induced drag coefficient $C_{D_{j}}$ was predicted theoretically using the vortex-lattice codes.

The relative advantages of the winglets cannot be realized at zero lift, since the configuration with winglets produces additional profile drag. However, as lift increases, the reduction in induced drag caused by the winglets begins to take effect. At lift coefficients beyond 0.4 , the configuration with winglets produces less total drag.

Table 1 presents the experimental and theoretical efficiency factors for the various biplane configurations. Theoretical values were calculated using the vortexlattice codes. Experimental values were determined by first subtracting the incremental profile drag coefficients of reference 9 from the measured drag, and then plotting them in the form of $C_{L}^{2}$ versus $C_{D}$. The efficiency factors were calculated from the slopes of the $C_{L}^{2}$ versus $C_{D}$.

It is common in biplane theory to calculate efficiency factors based on the biplane equivalent monoplane aspect ratio (EMAR), which is 3.38 for the configuration tested. The EMAR is a theoretical aspect ratio calculated for the biplane using reference 16. This calculation, which takes into account Prandtl's biplane interference factor, assumes equal and optimal load distributions on both biplane wings. Recause of the assumptions used in deriving EMAR (ref. 16), it is only a crude measure of relative efficiency; however, it is used herein since no alternate monopilane data was obtained.

For $0^{\circ}$ decalage, the theoretical efficiency factors were 1.091 and .974 , with and without winglets, respectively. Thus, the addition of winglets caused an increase in the theoretical efficiency factor of 12.0 percent. The experimental efficiency factors were determined using algebraic linear regression. They were .991 and .870; thus, a 13.9-percent increase was obtained experimentally.

For the $-5^{\circ}$ decalage case, the theoretical efficiency factors were found to be 1.083 and 1.166, an increase due to the winglet of 7.7 percent. Experimentally, the values were .831 and .981 , a difference of 12.8 percent. Therefore, based on experimental results, the addition of winglets increased the efficiency by approximately 13 percent.

The predicted induced drag is shown in figures 6 and 7 . It is of interest to note that the winglet effect is more pronounced for the $0^{\circ}$ decalage case than for the $-5^{\circ}$ decalage case.

Parametric Study.- Additional theoretical studies were made to study the effect of various winglet geometries on the biplane lifting efficiency and performance. Figure 8 shows the effect of winglet spanwise location on lifting efficiency. The winglet appears to have little effect unless it is located very close to the wing tip. Relative differences in efficiency between the $0^{\circ}$ and $-5^{\circ}$ decalage cases appear negligible. 
Figure 9 shows the effect of winglet twist. The winglet is toed in (at the maximum twist angle) at the upper wing-winglet intersection, and toed out (in the same manner) at the lower wing-winglet intersection, with a linear variation of twist occurring in between. For both decalage cases; the optimum twist appears to be zero.

Figure 10 shows the effect of decreasing the winglet planform area. Planform area was decreased by stripping away constant chord sections of area at the leading edge of the winglet. The winglet maintains its effectiveness quite well until approximately 70 percent of its original area is removed. Therefore, in order to minimize skin friction drag, the winglet should be resized to approximately 30 percent of its original chord.

Figures 11 and 12 show a theoretical comparison of the aerodynamic performance characteristics of the monoplane and biplane configurations. The biplane utilizes the resized winglet, which has been reduced to 30 percent of its original size in order to obtain good lifting efficiency while minimizing skin friction drag. A1] configurations were analyzed on the grounds of equal planform area and equivalent aspect ratios. Viscous drag was predicted using the equivalent parasite area method of reference 15. Inviscid drag was computed using the previously discussed potential flow codes.

Overall, the results indicate improved performance for the biplane. The nowinglet biplane showed a 5.4-percent increase in $L / D_{\max }$, while the biplane with winglet.s showed a 6.4-percent increase. The most significant performance improvement, however, was in the classical endurance parameter $C_{L}^{3 / 2} / C_{D}$. The no-winglet biplane showed an 8.1-percent increase in $c_{b}^{3 / 2} / C_{D}$ over the monoplane, while the biplane with winglets showed a significant 12.9-percent increase.

\section{CONCLUSIONS}

This study of the effects of winglets applied to biplanes shows that:

1. Theoretical computations are in good agreement with experiment in predicting inviscid drag due to lift.

2. Theoretical and experimental results indicate that the addition of winglets to an optimized biplane configuration can increase the ideal efficiency factor by up to 13 percent, as well as increasing the lift-curve slope and maximum lift coefficient.

3. A theoretical analysis comparing the biplane with an optimized winglet to an equivalent monoplane indicates that the biplane has the potential for a 6.4-percent increase in $L / D_{\max }$ and a 13 percent increase in $C_{L} 3 / 2 / C_{D}$, the classical endurance parameter. 


\section{REFERENCES}

1. Heyson, Harry H.; Riebe, Gregory D.; and Fulton, Cynthia L.: Theoretical Parametric Study of the Relative Advantages of Winglets and Wing-Tip Extensions. NASA TP-1020, 1977.

2. Whitcomb, Richard T.: A Design Approach and Selected Wind-Tunnel Results at High Subsonic Speeds for Wing-Tip Mounted Winglets. NASA TN D-8260, 1976.

3. Prandt1, L.: Induced Drag of Multiplanes. NACA TN-182, 1924.

4. Munk, Max, M.: General Biplane Theory. NACA Report 151, 1922.

5. Knight, Montgomery; and Noyes, Richard W.: Wind Tunnel Tests on a Series of Biplane Wing Models, Part I. Effects of Changes in Stagger and Gap. NACA TN 310, 1929.

6. Knight, Montgomery; and Noyes, Richard W.: Wind Tunnel Tests on a Series of Biplane Wing Models, Part II. Effects of Changes in Decalage, Dihedral, Sweepback, and Overhang. NACA TN 325, 1929.

7. Knight, Montgomery; and Noyes, Richard W.: Wind Tunnel Tests on a Series of Biplane Wing Models, Part III. Effects of Changes in Various Combinations of Stagger, Gap, Decalage, and Sweepback. NACA TN 330, 1929.

8. Norton, F. H.: The Effect of Staggering a Biplane. NACA TN 70, 1921.

9. Nenadovitch, Miroslave: Recherches Sur Les Cellules Biplanes Rigides D'Envergure Infine. Publications Scientifigues of Technigues du Ministere de L'Air, Institut Aerotechnigue de Saint-Cyr, Paris, 1936.

10. 01 son, E. C.; and Selberg, B. P.: Experimental Determination of Improved Aerodynamic Characteristics Utilizing Biplane Wing Configurations. Journal of Aircraft, Vol. 13, No. 4, 1974.

11. Rokhsaz, K.: Analytical Investigation of the Aerodynamic Characteristics of Dual Wing Systems. University of Missouri-Rolla Thesis, 1980.

12. Gall, Peter D.: An Experimental and Theoretical Analysis of the Aerodynamic Characteristics of a Biplane-Winglet Configuration. M.S. Thesis, Pennsylvania State University, 1984 (also available as NASA TM 85815).

13. Tulinius, J.: Unified Subsonic, Transonic, and Supersonic NAR Vortex Lattice. TFD-72-523, North American Rockwel1, 1972.

14. Jacobs, Eastman N.; and Sherman, Albert: Airfoil Section Characteristics as Affected by Variations in Reynolds Number. NACA Report 586, 1939.

15. Roskam, Jan: Methods for Estimating Drag Polars of Subsonic Airplanes. Roskam Aviation and Engineering Corp., 1971, pp 2.1-2.2.

16. Jones, B.: Element of Practical Aerodynamics. Wiley, New York, 1950, pp 109-126. 
$\infty$

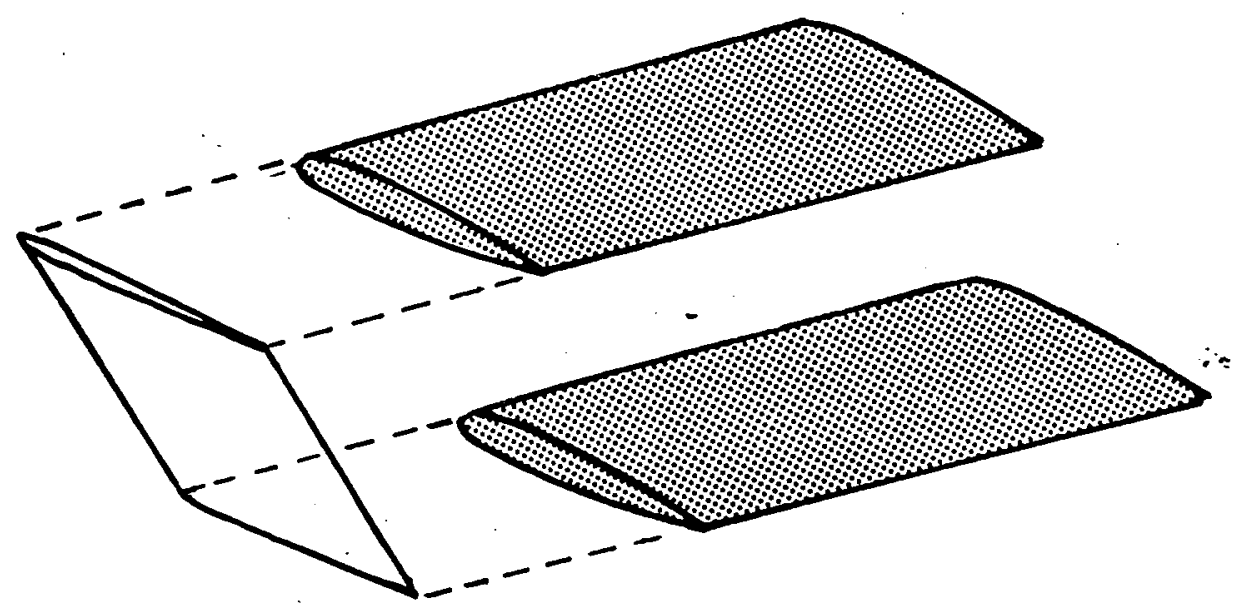

Figure 1.- Isometric view of model. 


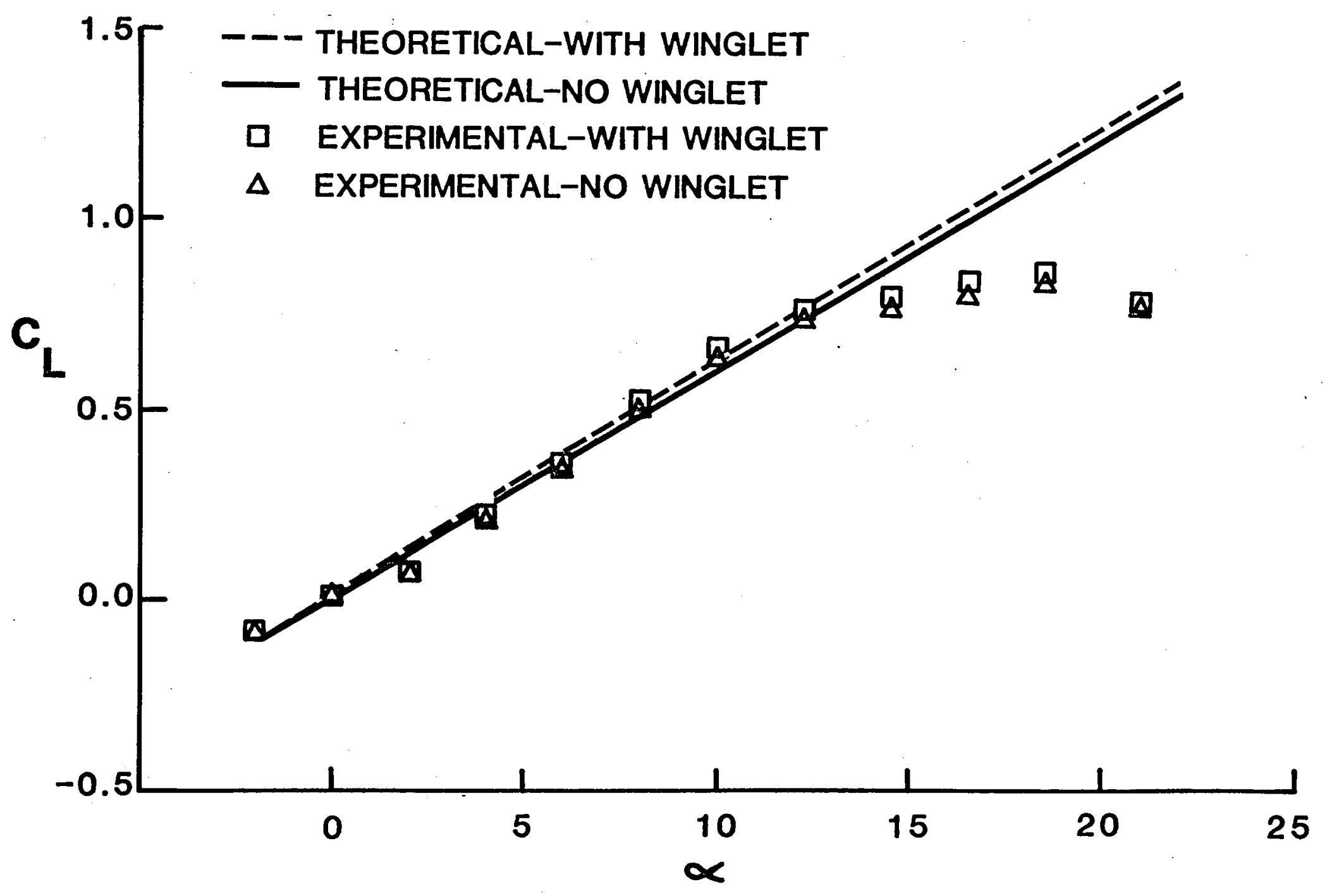




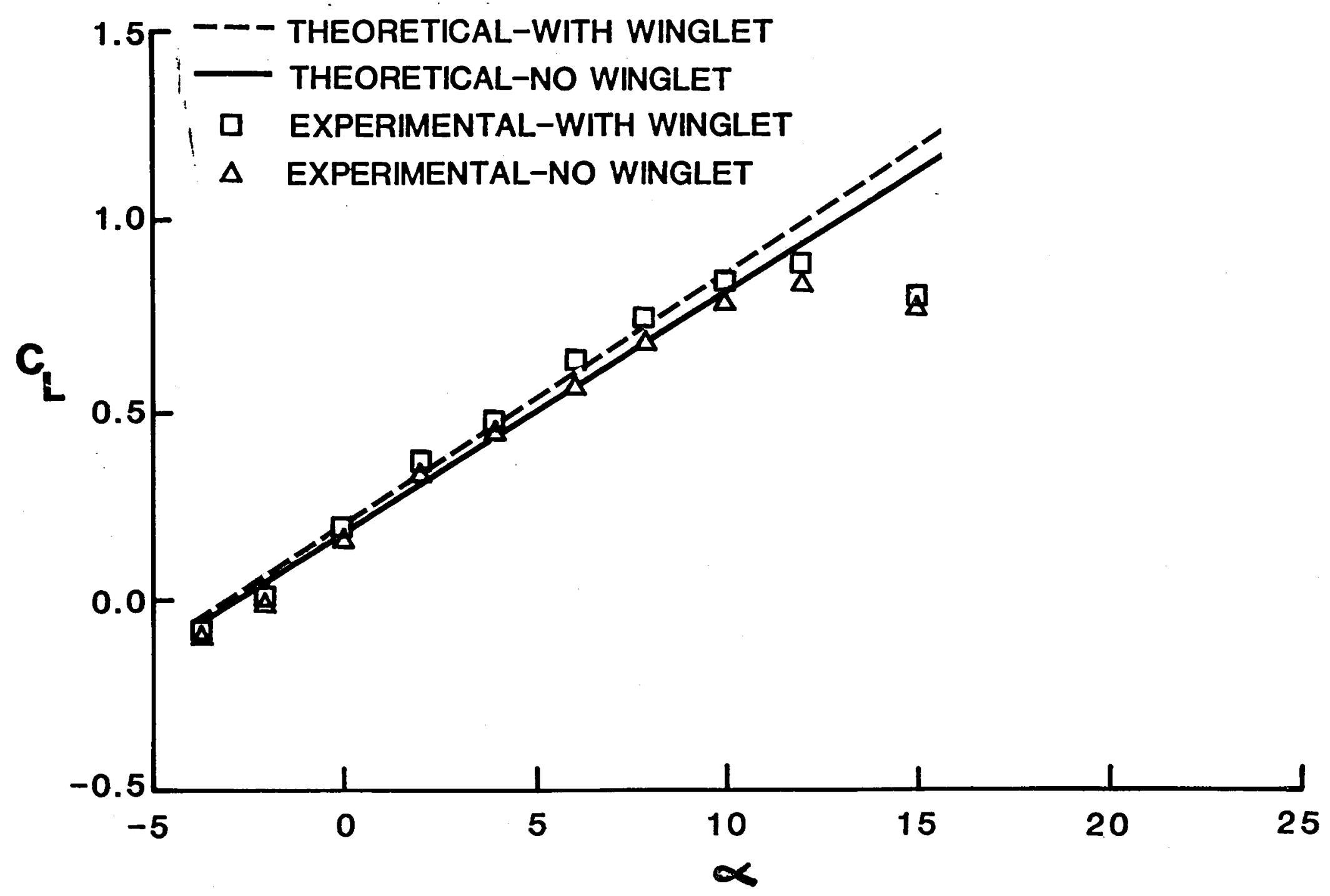

Figure 3.- Lift coefficient as function of angle of attack, $\mathrm{Ga}=1.0, \mathrm{St}=1.0, \mathrm{Dec}^{\circ}=-5^{\circ}$. 


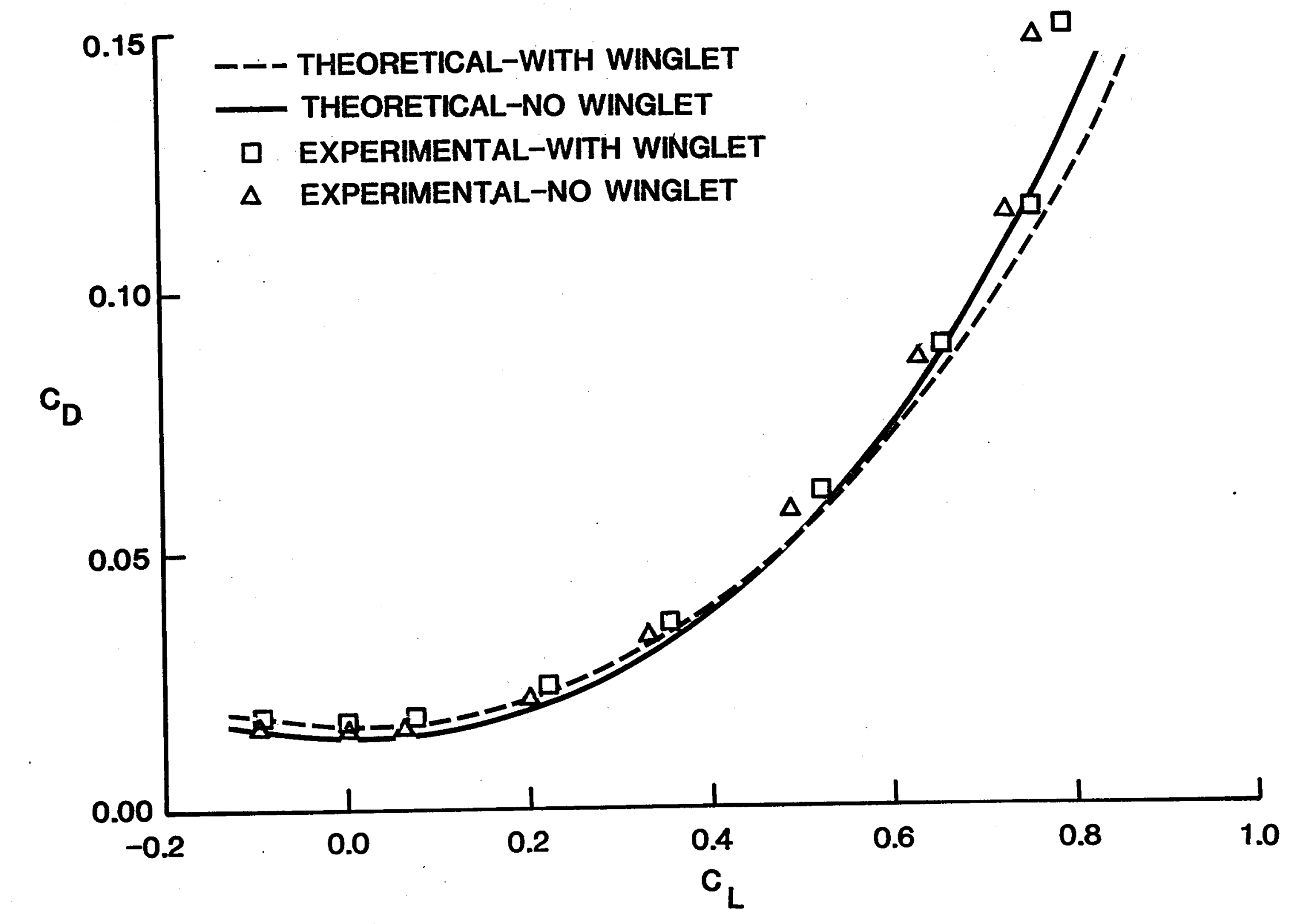

Figure 4.- Biplane drag polars, $\mathrm{Ga}=1.0, \mathrm{St}=1.0, \mathrm{Dec}=0^{\circ}$. 


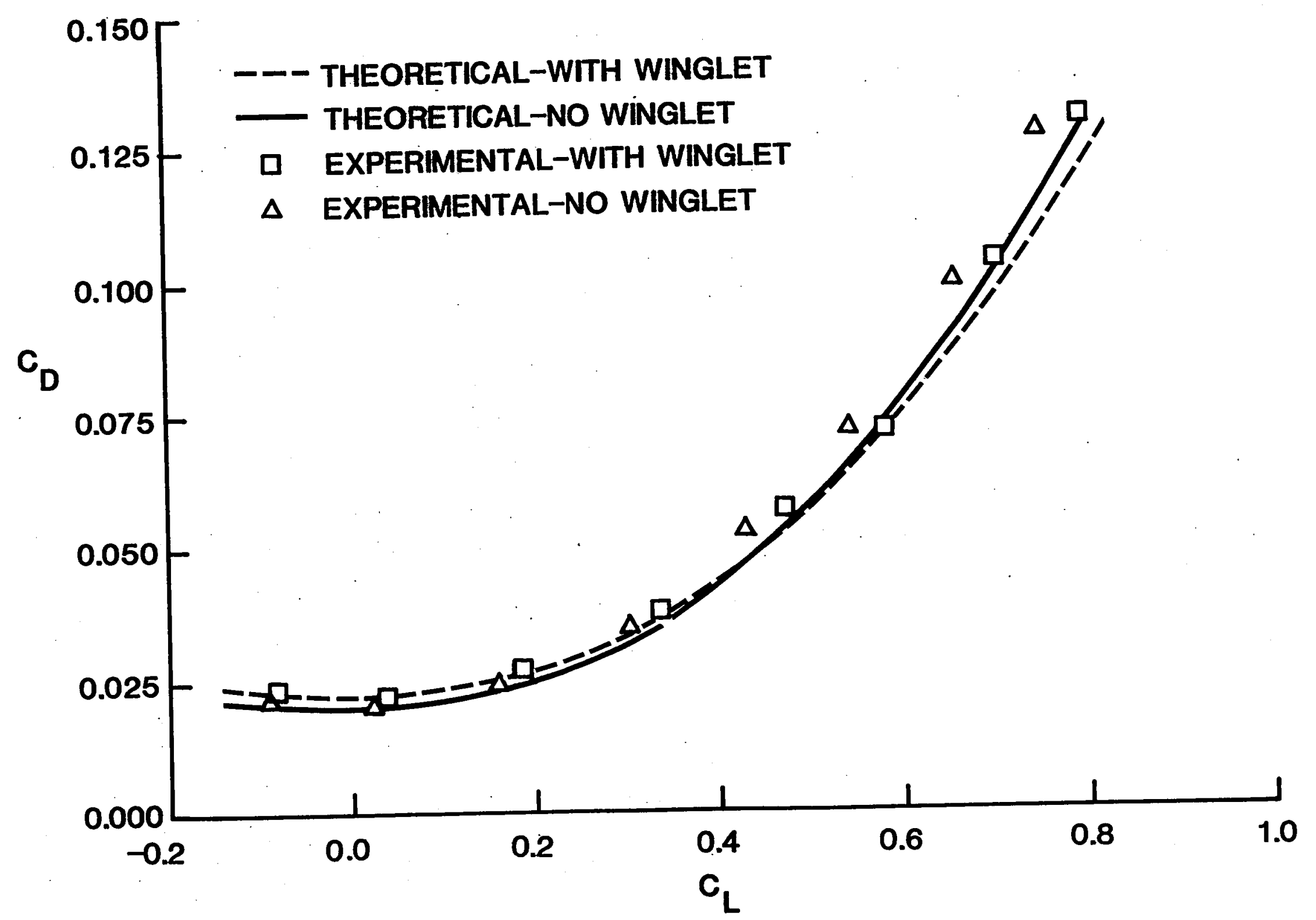

Figure 5.- Biplane drag polars, $G a=1.0, \mathrm{St}=1.0, \mathrm{DeC}=-5^{\circ}$. 


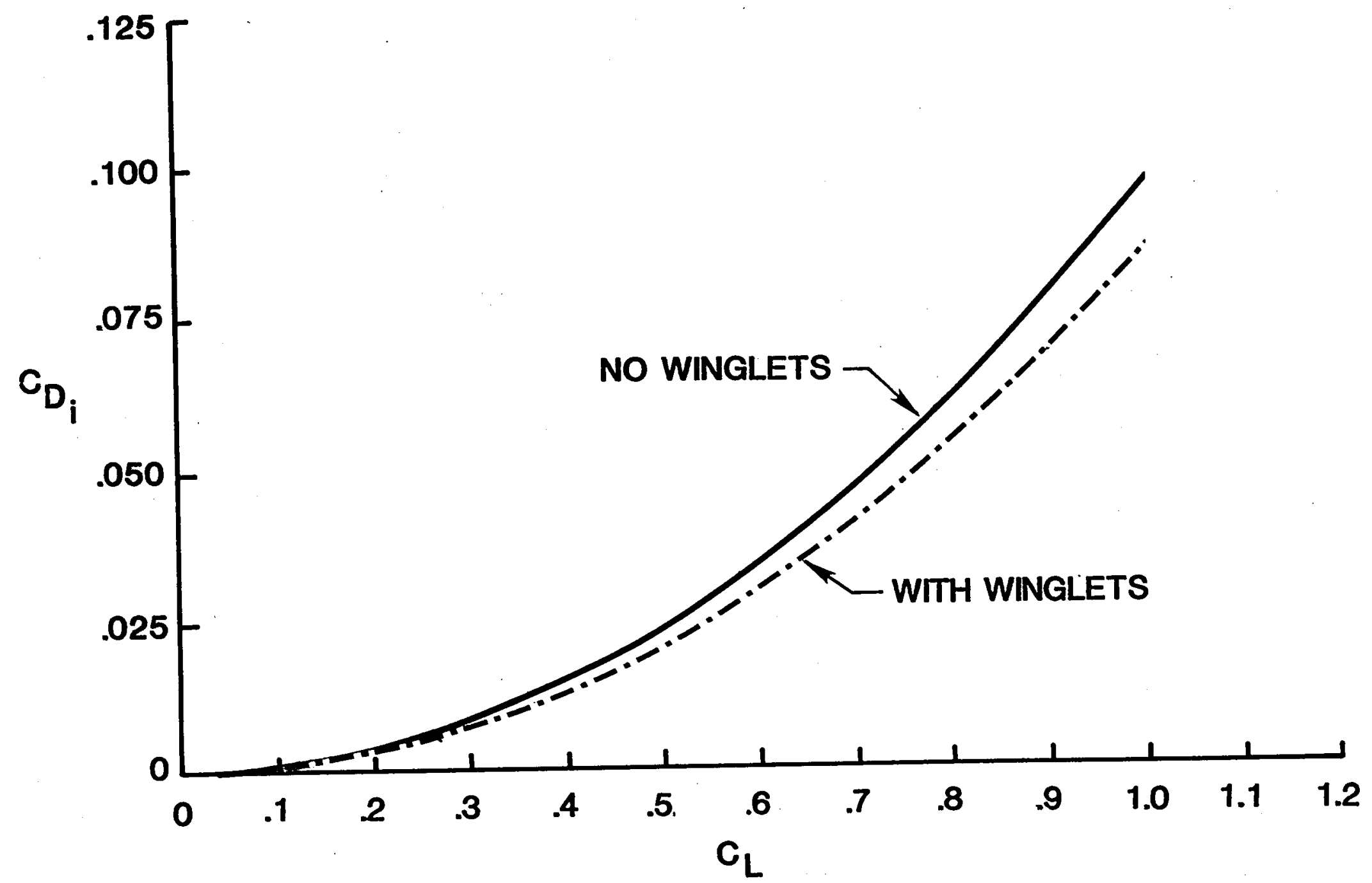




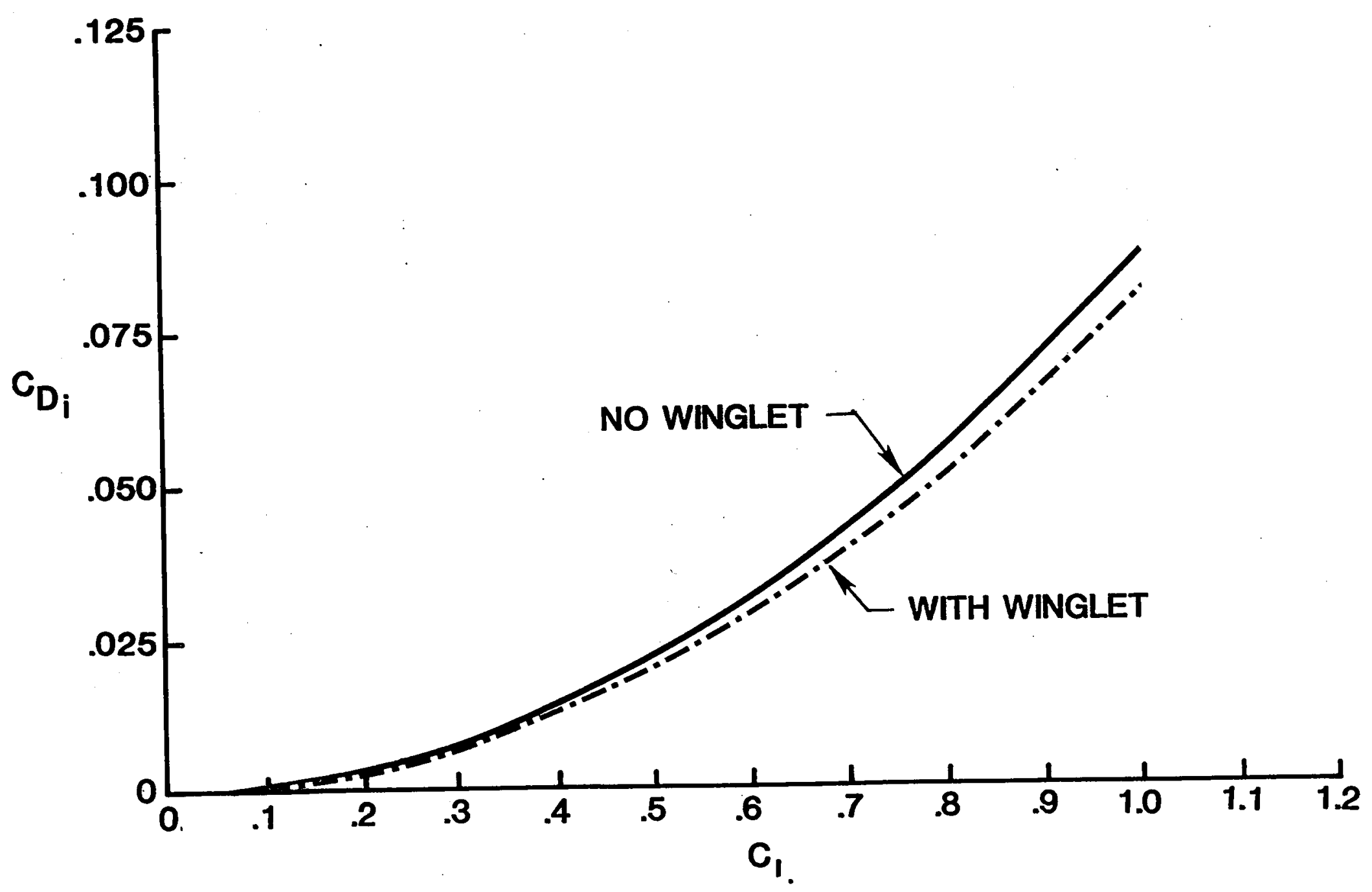

Figure 7.- Theoretically predicted induced drag, $\mathrm{Ga}=1.0, \mathrm{St}=1.0, \mathrm{Dec}=-5^{\circ}$. 


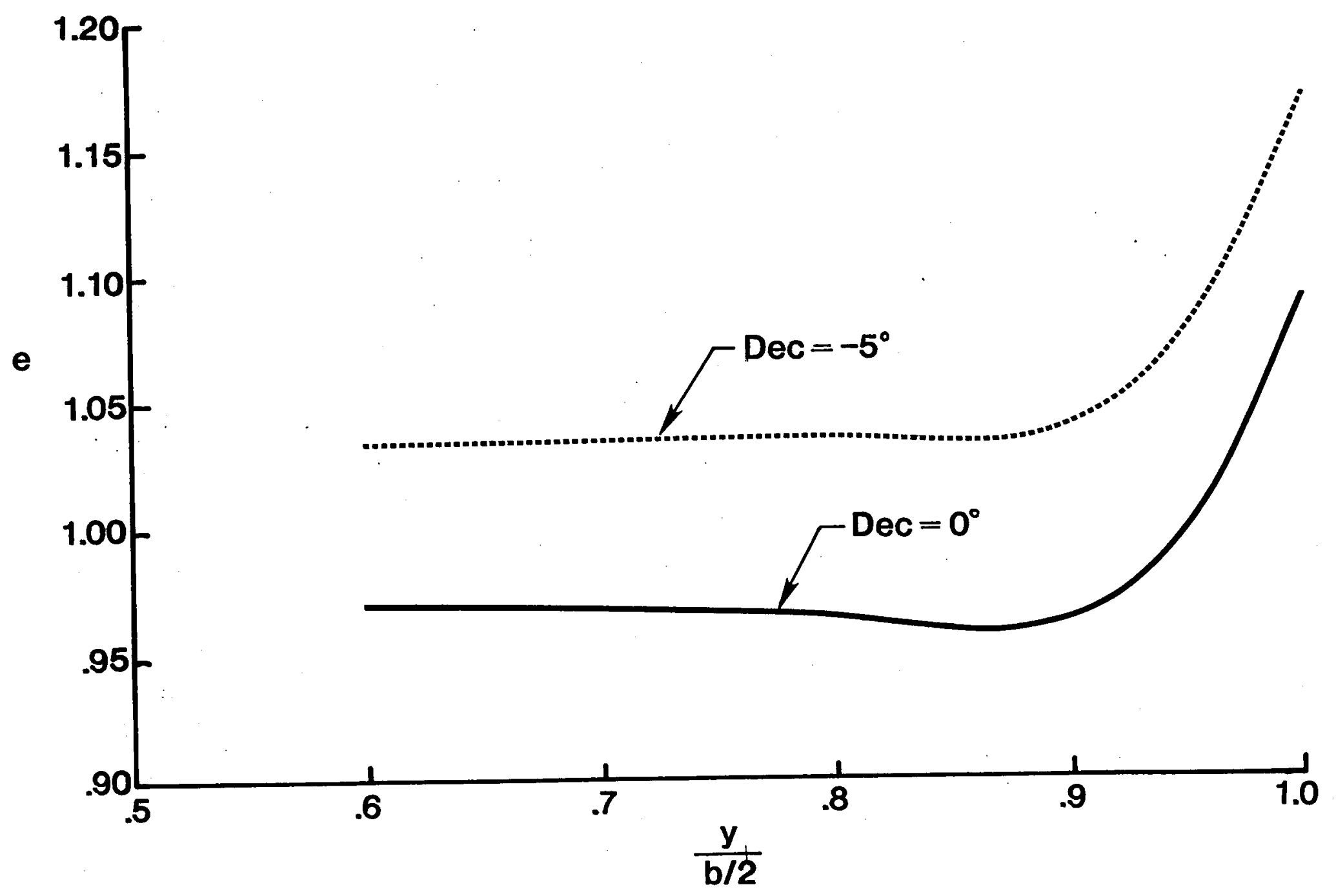




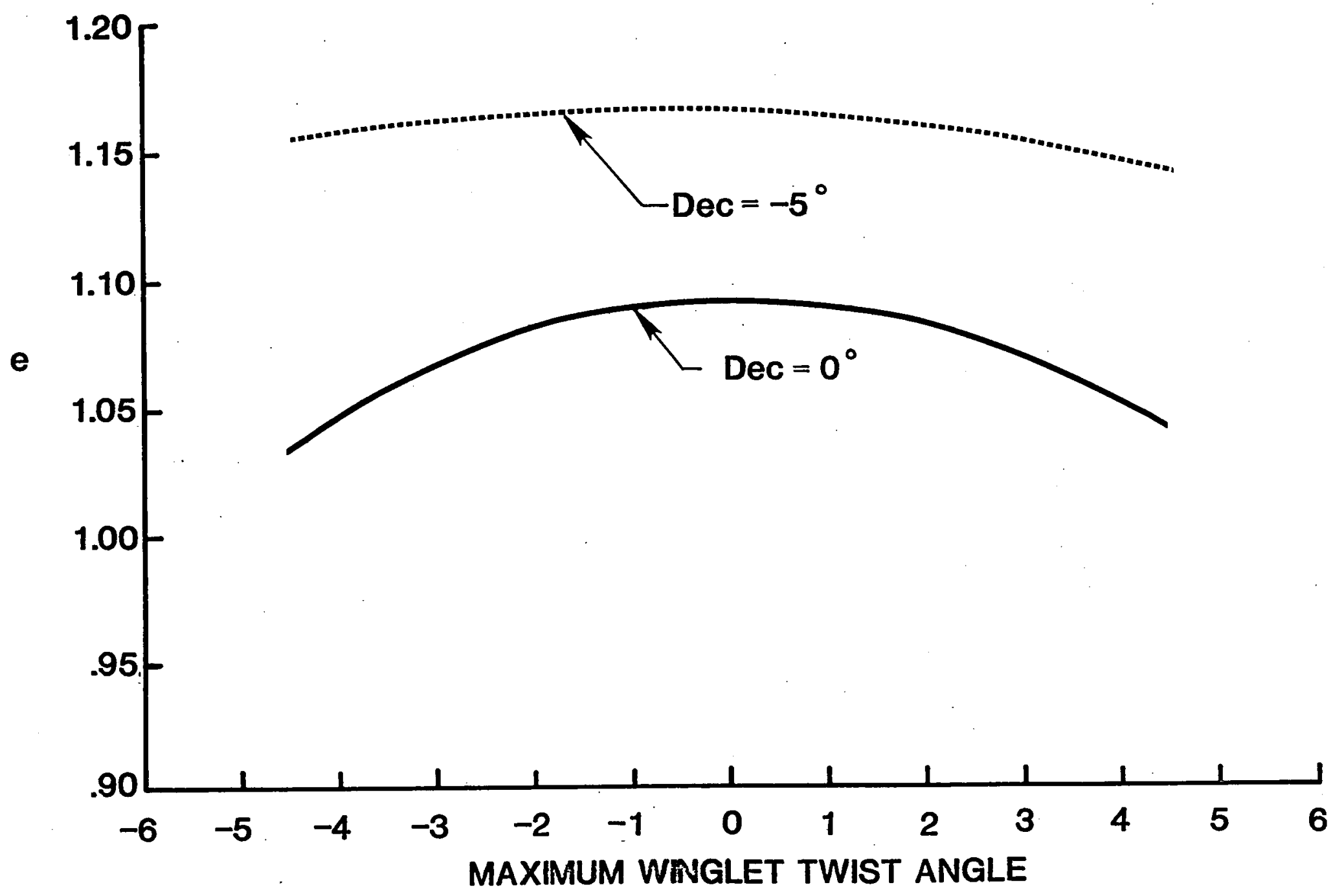

Figure 9.- Biplane efficiency as a function of winglet twist. 


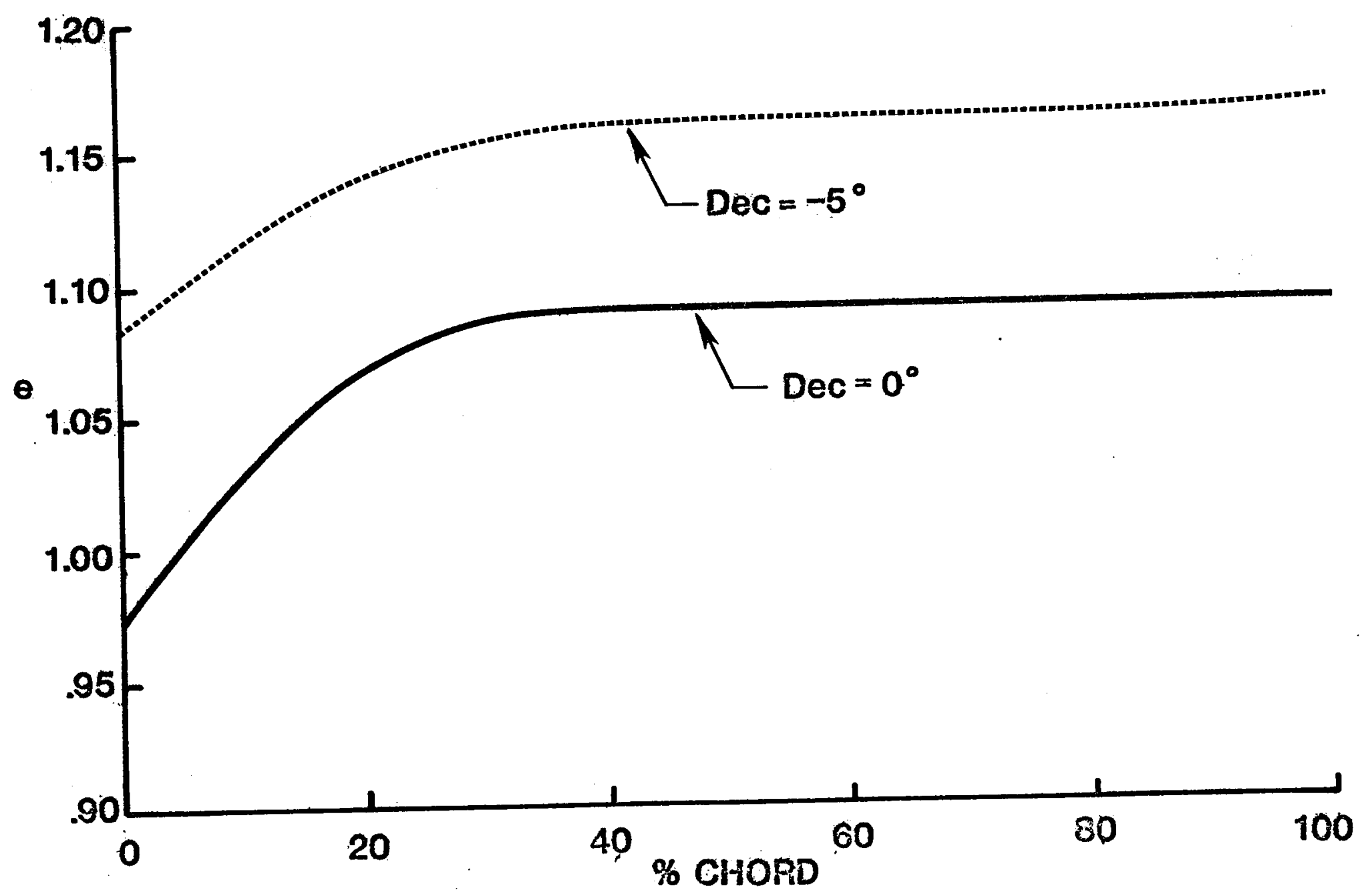




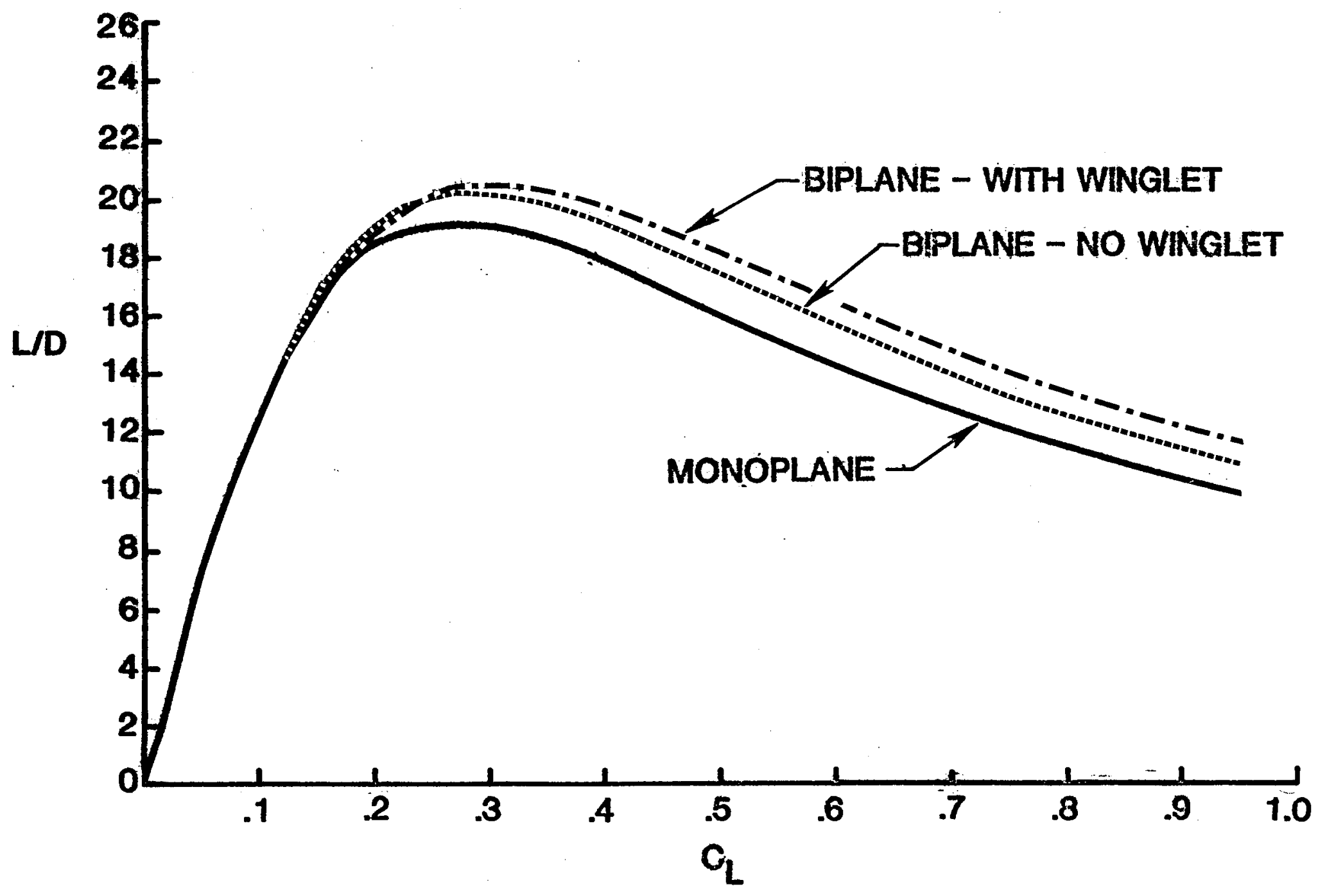

Figure 11.- Biplane and monoplane lift-drag ratios, $\mathbf{G a}=1.0$, St $=1.0$, Dec $=-5^{\circ}$. 


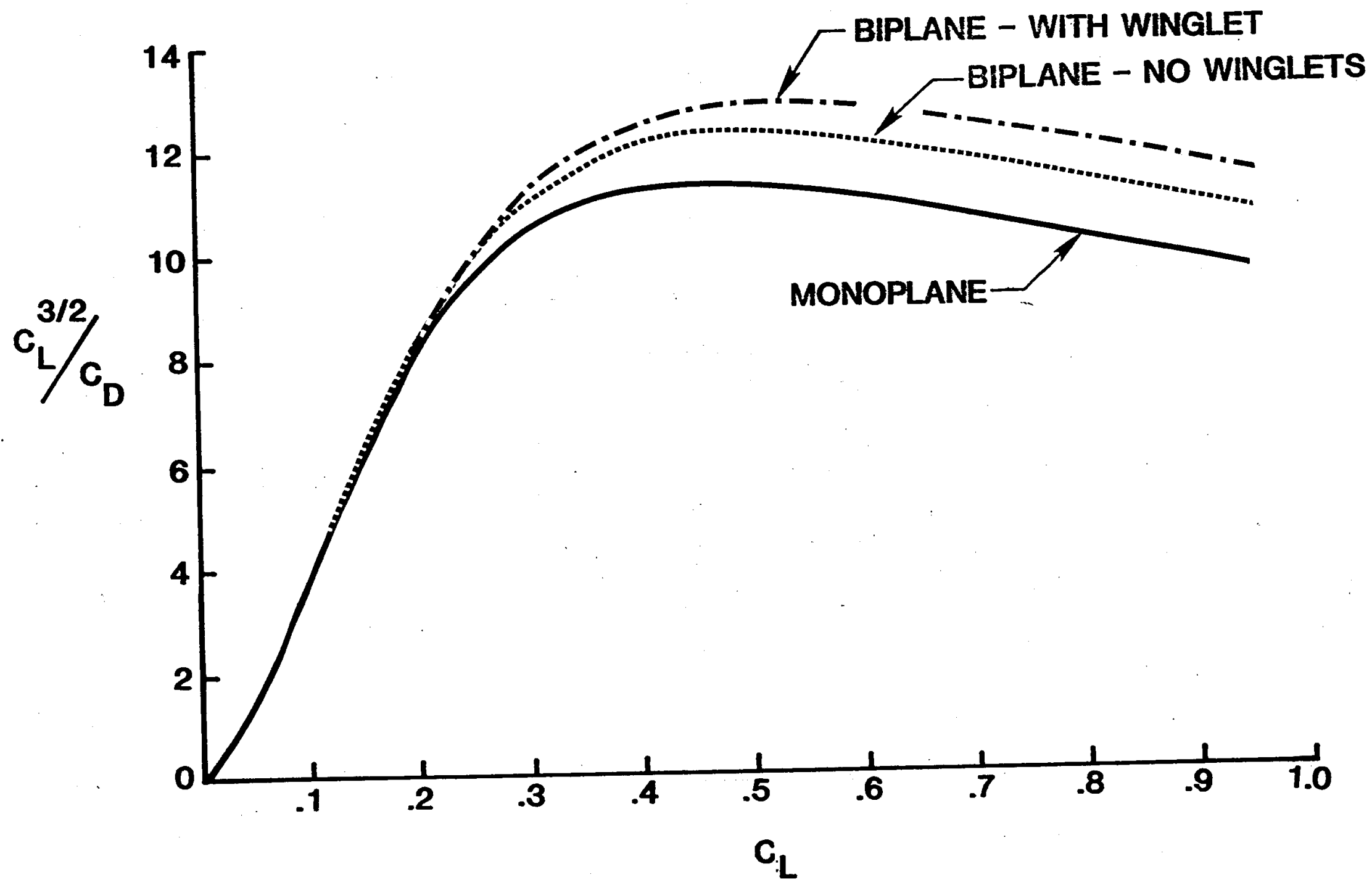




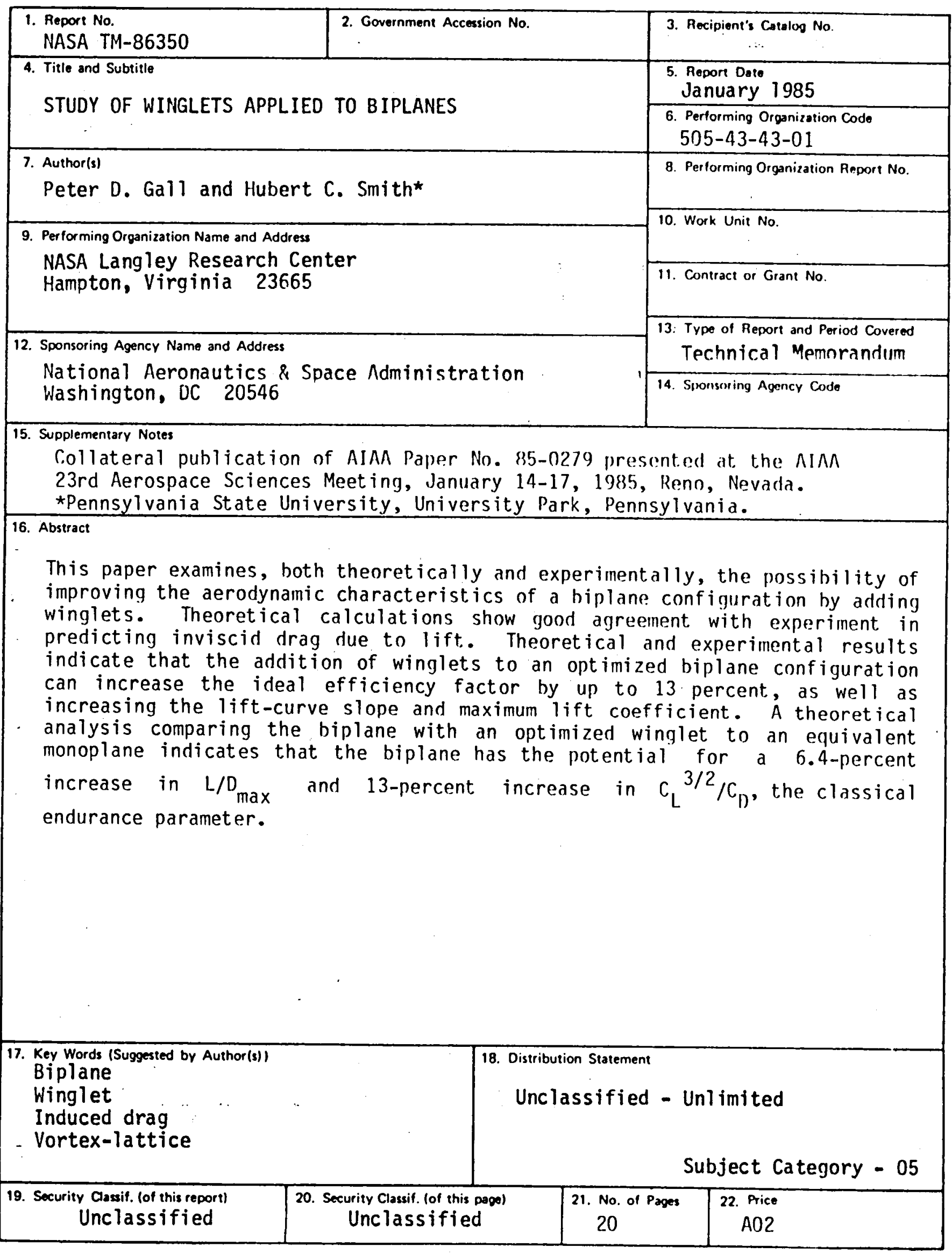




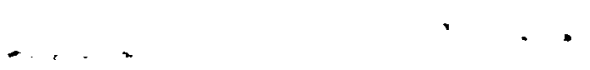




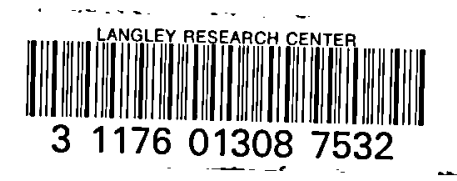

○ 